\title{
Recent developments in the radiation belt environment model
}

\author{
M.-C. Fok ${ }^{\mathrm{a}, *}$, A. Glocer ${ }^{\mathrm{a}}$, Q. Zheng ${ }^{\mathrm{a}, \mathrm{b}}$, R.B. Horne ${ }^{\mathrm{c}}$, N.P. Meredith ${ }^{\mathrm{c}}$, J.M. Albert ${ }^{\mathrm{d}}$, T. Nagai ${ }^{\mathrm{e}}$ \\ a Geospace Physics Laboratory, NASA Goddard Space Flight Center, Greenbelt, MD, USA \\ ${ }^{\mathrm{b}}$ Department of Astronomy, University of Maryland, College Park, MD, USA \\ ${ }^{\text {c }}$ Physical Science Division, British Antarctic Survey, Cambridge, UK \\ d Air Force Research Laboratory, Hanscom, MA, USA \\ e Department of Earth and Planetary Sciences, Tokyo Institute of Technology, Tokyo, Japan
}

\section{A R T I C L E I N F O}

\section{Article history:}

Received 1 April 2010

Received in revised form

28 September 2010

Accepted 30 September 2010

\section{Keywords:}

Radiation belts

Storm and substorm

Wave-particle interactions

Space weather

\begin{abstract}
A B S T R A C T
The fluxes of energetic particles in the radiation belts are found to be strongly controlled by the solar wind conditions. In order to understand and predict the radiation particle intensities, we have developed a physics-based Radiation Belt Environment (RBE) model that considers the influences from the solar wind, ring current and plasmasphere. Recently, an improved calculation of wave-particle interactions has been incorporated. In particular, the model now includes cross diffusion in energy and pitch-angle. We find that the exclusion of cross diffusion could cause significant overestimation of electron flux enhancement during storm recovery. The RBE model is also connected to MHD fields so that the response of the radiation belts to fast variations in the global magnetosphere can be studied. We are able to reproduce the rapid flux increase during a substorm dipolarization on 4 September 2008. The timing is much shorter than the time scale of wave associated acceleration.
\end{abstract}

Published by Elsevier Ltd.

\section{Introduction}

The Earth's radiation belts consist of energetic electron $(\sim 100 \mathrm{keV}$ to several MeV) and ions ( $\sim 100 \mathrm{keV}$ to several hundred $\mathrm{MeV})$ trapped in the magnetosphere roughly from $1.2<L<8$. The energetic electrons reside in 2 distinct regions: the inner belt and the outer belt, which are usually separated by the slot region $(1.8<L<3)$ of depleted particle populations. Pitch-angle diffusion loss of electrons by interacting with whistler mode plasmaspheric hiss is believed to be the cause of the slot region (Lyons et al., 1972; Albert, 1994; Meredith et al., 2007). The inner belt is relatively stable while the outer belt is highly variable with geomagnetic activity. The fluxes of energetic electrons in the outer belt decrease during the main phase of a magnetic storm due to adiabatic effect (Dessler and Karplus, 1961; Kim and Chan, 1997). Additional non-adiabatic processes also contribute to the flux decrease in the storm main phase (Green et al., 2004; Ukhorskiy et al., 2006). During the recovery phase the flux of energetic electrons can change dramatically as well. While approximately half of all moderate and intense storms cause a net increase in the flux of energetic electrons by a factor of 2 or more, approximately a quarter of these storms result in a net decrease in the fluxes by more than a factor of 2 (Reeves et al., 2003). This variability is caused by an imbalance between acceleration, transport, and loss processes all of which become enhanced during geomagnetic storms (Horne, 2002; Thorne et al., 2005; Horne et al., 2006; Summers et al., 2007). The ratio of post-storm to pre-storm flux

\footnotetext{
* Corresponding author. Tel.: +1 3012861083.

E-mail address: mei-ching.h.fok@nasa.gov (M.-C. Fok).
}

was found related to the solar wind speed (Paulikas and Blake, 1979; Reeves et al., 2003) and the direction of the IMF Bz during the storm recovery phase (Iles et al., 2002). On the other hand, Ukhorskiy and Sitnov (2008) suggested that the outer belt can respond differently to similar solar wind driving. There is a broad range of processes that shape the radiation belts. Some of them are nonlinear mechanisms of local particle acceleration, such as, interactions with whistler mode chorus waves (Summers et al., 2004; Omura and Summers, 2006), and relativistic electrons drift-resonance with large amplitude, narrow bandwidth Pc 5 waves (Degeling et al., 2008).

The intensification of the radiation belts has significant space weather consequences. Moderate energy ( $\sim 10-100 \mathrm{keV})$ electrons can cause surface charging effects and relativistic $(\sim 0.1-5 \mathrm{MeV})$ electrons can cause deep-dielectric charging on space systems (Baker, 2001). Therefore, understanding the physical processes that are controlling the development of the radiation belts during active periods and being able to predict their variability have both scientific and practical significance.

There exist multiple sources of radiation belt particles. Radial diffusion has traditionally been considered to be the leading transport and energization mechanism in the inner magnetosphere (Schulz and Lanzerotti, 1974). However, it has recently been suggested that electrons can be accelerated efficiently by resonant wave-particle interactions with whistler mode chorus waves (Horne and Thorne, 1998; Summers et al., 1998) and fast magnetosonic waves (Horne et al., 2007).

A number of kinetic models have been established to simulate the radiation belt dynamics and to provide interpretation for observable features. In a kinetic model, the equation for the particle 
distribution function is solved analytically or numerically. One simple approach is based on a standard radial diffusion equation with diffusion coefficients driven by the solar wind conditions or geomagnetic activity (Li et al., 2001; Albert et al., 2001). Varotsou et al. (2005) and Horne et al. (2006) combined radial diffusion with acceleration and loss due to whistler mode chorus waves and confirmed that wave acceleration by whistler mode chorus is an important acceleration mechanism in the outer radiation belt. By a similar approach but including cross diffusion terms due to chorus waves, Albert et al. (2009) simulated the 9 October 1990 magnetic storm. They found both chorus wave acceleration and radial diffusion were required to account for the observed increase in relativistic electron flux during the recovery phase. For models that cover a wide range of energy, drift motion must be considered since convection is an important transport mechanism for lower-energy $(<50 \mathrm{keV}$ ) particles (Bourdarie et al., 1997; Zheng et al., 2003; Miyoshi et al., 2006; Fok et al., 2008). Using the relativistic kinetic model with dipole magnetic field, Miyoshi et al. (2006) simulated the dynamics of energetic electrons during the October 2001 storm. They reproduced the observed local time flux asymmetry for hot electrons $(30 \mathrm{keV})$. They also concluded that only convective transport and radial diffusion cannot explain the enhancement of relativistic electrons seen during storm recovery.

A convection-diffusion model, namely the Radiation Belt Environment (RBE) model, has been developed to understand the radiation belt dynamics in order to predict the flux variation during active times (Fok et al., 2001, 2005, 2008; Zheng et al., 2003). The RBE model employs time-varying, realistic magnetic field so that radial diffusion effects due to slow magnetic fluctuations and inductive radial transport owing to fast magnetic reconfiguration can be modeled. The RBE model was used to simulate a substorm injection during a dipolarization of the magnetic field (Fok et al., 2001). Observable features during substorms, such as dispersionless injection and drift echoes, are successfully reproduced. Electron flux enhancements during magnetic storms were also studied using the RBE model (Zheng et al., 2003; Fok et al., 2005, 2008). They found that energization by the inductive electric field and by whistler mode waves is crucial for the flux increase during magnetic storms. A simplified version of the RBE model is currently running in real-time to provide radiation belt now-casting updated every $15 \mathrm{~min}$. The geosynchronous fluxes at longitudes of GOES-11 and 13 are extracted from the RBE real-time run and are plotted together with real-time GOES electron $(>0.6 \mathrm{MeV})$ data. The model-data comparison is continually posted at http://mcf.gsfc.nasa.gov/RB_nowcast/.

Recent developments, discussed in this paper, expand on past work in a number of ways. In previous RBE simulations of waveparticle interactions, only pure energy and pure pitch-diffusion are considered (Fok et al., 2005, 2008). Since cross diffusion may have a comparable effect to pure diffusions (Albert and Young, 2005), the RBE model has been extended to include cross diffusion in energy and pitch-angle. We choose to use the Alternating Direction Implicit (ADI) method (Xiao et al., 2009) to solve the cross diffusion terms in the RBE equation. We revisit the geomagnetic storm on 23-27 October 2002 and quantitatively assess the influence of cross diffusion in electron flux enhancement during the recovery phase. Another development in the RBE model is connecting the model with a global magnetohydrodynamic (MHD) model. Glocer et al. (2009) coupled the RBE model with the BlockAdaptive-Tree-Solarwind-Roe-Upwind-Scheme (BATSRUS) MHD model to simulate radiation belt development during steady solar wind conditions and during a real storm event. They found the coupled code was able to create rapid inward transport on the time scale of tens of minutes. In this work, we use the RBE-BATSRUS code to simulate a substorm injection during the 3-5 September 2008 storm. We examine the timing and location of electron enhancement during the dipolarization event. In the following, a brief description of the RBE model is given. We then outline the algorithm of the ADI method and present the simulation results with cross diffusion included. Finally we present the RBE simulations of a substorm dipolarization and compare the calculations with Akebono electron data.

\section{Radiation belt environment (RBE) model}

The RBE model is a kinetic model that solves the bounceaveraged Boltzmann equation to obtain the distribution function of energetic electrons (Fok et al., 2008)

$$
\begin{aligned}
\frac{\partial f}{\partial t}+ & \left\langle\dot{\lambda}_{i}\right\rangle \frac{\partial f}{\partial \lambda_{i}}+\left\langle\dot{\phi}_{i}\right\rangle \frac{\partial f}{\partial \phi_{i}} \\
= & \frac{1}{G} \frac{\partial}{\partial \alpha_{o}}\left[G\left(D_{\alpha_{0} \alpha_{o}} \frac{\partial f}{\partial \alpha_{o}}+D_{\alpha_{0} E} \frac{\partial f}{\partial E}\right)\right] \\
& +\frac{1}{G} \frac{\partial}{\partial E}\left[G\left(D_{E E} \frac{\partial f}{\partial E}+D_{E \alpha_{o}} \frac{\partial f}{\partial \alpha_{o}}\right)\right]-\left(\frac{f}{0.5 \tau_{b}}\right)_{\text {loss cone }}
\end{aligned}
$$

where

$G=T\left(\alpha_{o}\right) \sin 2 \alpha_{o}\left(E+E_{o}\right) \sqrt{E\left(E+2 E_{o}\right)}$

$f=\left(t, \lambda_{I}, \phi_{I}, M, K\right)$, is the average distribution function on the field line between mirror points. $\lambda_{i}$ and $\phi_{i}$ are the magnetic latitude and local time, respectively, at the ionospheric foot point of the geomagnetic field line. $M$ is the relativistic magnetic moment and $K=J / \sqrt{8 m_{0} M}$, where $J$ is the second adiabatic invariant. The motion of the particles is described by their drifts across field lines, which are labeled by their ionospheric foot points. The inner boundary of $\lambda_{i}$ is at $11.8^{\circ}$, corresponding to $L=1.06$. The outer edge of the model is bounded by field lines with $\lambda_{i}$ not greater than $70.2^{\circ}$ and an equatorial crossing at 10 Earth radius $\left(R_{E}\right)$, whichever is closer. The $M$ range is chosen to fully represent the energies of electrons from $10 \mathrm{keV}$ to $6 \mathrm{MeV}$. The $K$ range is chosen to cover the loss cone so that particle precipitations can be estimated as well. $E_{o}$ is the electron rest energy, $\alpha_{o}$ is the equatorial pitch-angle, and $T\left(\alpha_{0}\right)$ is given by

$T\left(\alpha_{0}\right)=\frac{1}{R_{0}} \int_{0}^{s_{m}} \frac{d s}{\cos \alpha}$

where $R_{o}$ is the radial distance in $R_{E}$ of the field line equatorial crossing. The integration is along the field line from the equator to the mirror point. $\tau_{b}$ is the particle bounce period.

The left hand side of (1) represents the drifts of the particle population and the terms on the right hand side of (1) refer to diffusion and loss. The calculation of the bounce-averaged drift velocities across field lines, $\left\langle\dot{\lambda}_{i}\right\rangle$ and $\left\langle\dot{\phi}_{i}\right\rangle$, were described in detail in Fok and Moore (1997). These drifts include gradient and curvature drift, $\mathrm{E} \times \mathrm{B}$ drift from convection, and corotation electric fields. In this "drift" approach, the RBE model can simulate the effects of radial diffusion only if the electric and magnetic field fluctuations are properly and consistently represented.

The effects of the inductive electric field due to a time-varying magnetic field are also taken into account implicitly in the model (Fok et al., 2005). We have assumed that field lines are rooted at the ionosphere, so that the inductive electric field there is zero. However, the shapes of field lines at higher altitudes vary as a function of time according to the magnetic field model. If field lines are perfect conductors, the field line motion at high altitudes, e.g., at the equator, will generate an induction electric field $\left(\mathbf{E}_{\text {ind }}\right)$ of the form,

$\mathbf{E}_{\text {ind }}=-\mathbf{v}_{o} \times \mathbf{B}_{o}$ 


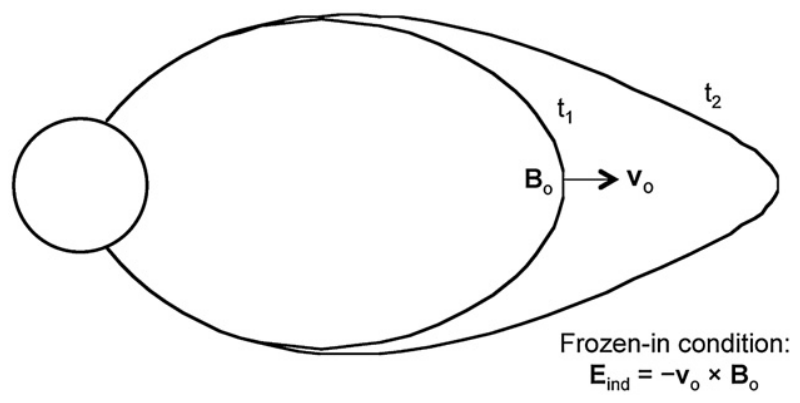

Fig. 1. An illustration to show the generation of the inductive electric field ( $\left.\mathbf{E}_{\text {ind }}\right)$ during field-line re-configuration.

where $\mathbf{v}_{o}$ and $\mathbf{B}_{o}$ are the field line velocity and magnetic field at the equator. Fig. 1 is an illustration on field line mapping changing with time and the generation of $\mathbf{E}_{\text {ind }}$. Under the frozen-in condition, particles initially moving along a particular field line at $t_{1}$ will continue to share the same field line at $t_{2}$ when the field line becomes more stretched, as shown in Fig. 1. Particles are drifting outward and decelerated in this case, and the first and second adiabatic invariants are conserved if the field line motion is slow. In the RBE model, electrons are energized or de-energized during the course of varying the magnetic configuration. The electron pitch angles will change as well.

The first two terms on the right hand side of (1) represent particle diffusion in energy and pitch-angle from interactions with plasma waves. When solving these two terms, we first map the particle phase space density from $(M, K)$ to $\left(E, \alpha_{o}\right)$ coordinates, perform diffusion in $E$ and $a_{\mathrm{o}}$, and then map the updated distribution back to the $(M, K)$ coordinates (Fok et al., 1996). The diffusion terms are followed by losses due to the loss cone, the boundary of which is assumed to correspond to mirror height of $120 \mathrm{~km}$. Particles in the loss cone are assumed to have a lifetime of one half bounce period $\left(0.5 \tau_{b}\right)$ (Lyons, 1973).

Eq. (1) includes multiple processes of different timescales. We use the method of fractional step or operator splitting to decompose the equation and solve only one term at a fractional step (Fok et al., 1993). To solve (1), we have to specify the electric and magnetic fields, the initial distribution, and the particle distribution on the nightside boundary, which is set at $10 R_{E}$ or the last closed field line. The effect of radial diffusion is incorporated via these time-varying electric and magnetic fields. The NASA trapped radiation model (AE8MAX) (Vette, 1991; Fung, 1996) is used for the initial condition in the entire RBE spatial domain. The distribution at the nightside boundary is assumed to be a kappa function with density $\left(N_{p s}\right)$ and characteristic energy $\left(E_{p s}\right)$ modeled by linear relations with the upstream solar wind conditions (Zheng et al., 2003)

$N_{p s}(t)=\left[0.02 N_{s w}(t-2 \mathrm{~h})+0.316\right] \sqrt{\mathrm{amu}}$

$E_{p s}(t)=0.016 V_{s w}(t-2 \mathrm{~h})-2.4$

where $N_{p s}$ is in $\mathrm{cm}^{-3}, N_{s w}$ is the solar wind density in the same unit, amu is the atomic mass unit of the electron, $E_{p s}$ is in $\mathrm{keV}$, and $V_{s w}$ is the solar wind velocity in $\mathrm{km} / \mathrm{s}$. Note that we assume a $2 \mathrm{~h}$ time lag between the plasma sheet condition and solar wind condition at the dayside magnetopause (Borovsky et al., 1998).

\section{Cross diffusion in the RBE model}

We have previously simulated the evolution of radiation belt electrons during the 23-27 October 2002 geomagnetic storm using the RBE model (Fok et al., 2008). The magnetic field is specified by the Tsyganenko 2004 (T04) model (Tsyganenko et al., 2003) and electric field by Weimer 2000 model (Weimer, 2001). Magnetic and electric fields are updated every $5 \mathrm{~min}$ according to the instantaneous solar wind condition and Dst index. The bounceaveraged diffusion coefficients are given by the Pitch-Angle and Energy Diffusion of Ions and Electrons (PADIE) code (Glauert and Horne, 2005). Only resonance with lower-band whistler mode chorus $\left(0.1 f_{c e}<f<0.5 f_{c e}\right)$ is considered. The presence of chorus waves is confined between $-15^{\circ}$ and $15^{\circ}$ magnetic latitude. The diffusion coefficients are calculated as a function of $L$ shell, energy, pitch-angle and $f_{p e} / f_{c e}$, the ratio of plasma frequency to the cyclotron frequency. To calculate $f_{p e} / f_{c e}$, which depends on the plasma density $\left(n_{e}\right)$, we have embedded inside the RBE model the core plasma model of Ober and Gallagher (Ober et al., 1997). The PADIE diffusion coefficients are scaled with a chorus wave intensity of $10^{4} \mathrm{pT}^{2}$. To obtain the actual diffusion coefficients, we estimate the chorus intensity at a given location and time during the storm using the survey of CRRES plasma wave data for lowerband chorus presented by Meredith et al. (2001, 2003). For our application the wave data were binned in $L$ shell, magnetic local time and 3 levels of magnetic activity $(K p<2 ; 2 \leq K p<4 ; K p \geq 4)$.

In Fok et al. (2008), we only considered pure energy and pure pitch-angle diffusion. Recently, we have implemented cross diffusion in the RBE model. The cross diffusion terms can be solved using various methods or numerical schemes (Albert and Young, 2005; Tao et al., 2008; Xiao et al., 2009; Shprits et al., 2009). We choose the Alternating Direction Implicit (ADI) method, which we found is stable, efficient and easy to implement. In the ADI scheme, the cross diffusion terms in (1) are rewritten as (Xiao et al., 2009)

$$
\begin{aligned}
\frac{\partial f}{\partial t} & =\frac{1}{G} \frac{\partial}{\partial \alpha_{o}}\left(G D_{\alpha_{o} E} \frac{\partial f}{\partial E}\right)+\frac{1}{G} \frac{\partial}{\partial E}\left(G D_{E \alpha_{o}} \frac{\partial f}{\partial \alpha_{o}}\right) \\
& =\frac{1}{G} \frac{\partial(G D)}{\partial \alpha_{o}} \frac{\partial f}{\partial \varepsilon}+\frac{1}{G} \frac{\partial(G \tilde{D})}{\partial \varepsilon} \frac{\partial f}{\partial \alpha_{o}}+2 \tilde{D} \frac{\partial^{2} f}{\partial \alpha_{o} \partial \varepsilon} \\
& =\hat{I}_{1} f+\hat{I}_{2} f+\hat{I}_{3} f
\end{aligned}
$$

where

$$
\varepsilon=E / E_{o}, \tilde{D}=\varepsilon D_{E \alpha_{o}} / E=\varepsilon D_{\alpha_{0} E} / E
$$

The cross diffusion terms are thus expressed as derivative in $\varepsilon$ $\left(\hat{I}_{1}\right)$, derivative in $\alpha_{o}\left(\hat{I}_{2}\right)$ plus mix derivative in $\varepsilon$ and $\alpha_{o}\left(\hat{I}_{3}\right)$. To advance $f$ in time, a time step is divided into two sub-steps as

$f^{n+1 / 2}=f^{n}+\frac{\Delta t}{2}\left[\hat{I}_{1} f^{n+1 / 2}+\hat{I}_{2} f^{n}+\hat{I}_{3} f^{n}\right]$

$f^{n+1}=f^{n+1 / 2}+\frac{\Delta t}{2}\left[\hat{I}_{1} f^{n+1 / 2}+\hat{I}_{2} f^{n+1}+\hat{I}_{3} f^{n+1 / 2}\right]$

where

$f^{n}=f(t)$ at $t=n \Delta t$

In the first sub-step, $\hat{I}_{1}$ is solved implicitly and $\hat{I}_{2}$ explicitly. In turn, in the second sub-step, $\hat{I}_{2}$ is solved implicit and $\hat{I}_{1}$ explicitly. $\hat{I}_{3}$ is always solved explicitly. The finite difference representation of (6) is given in the Appendix.

Having previously simulated the energetic electron fluxes during the 23-27 October 2002 event (Fok et al., 2008), we revisit this event with cross diffusion terms included in the governing equation. Fig. 2 shows the $L$-time diagrams of equatorial electron fluxes in energy ranges of $20-70 \mathrm{keV}$ (left panels) and $0.6-1.8 \mathrm{MeV}$ (right panels); radial transport due to time-varying magnetic and electric fields are included in all three rows. The top panels of Fig. 2 display results in which wave diffusion is not included in the calculations. The Dst index (black curve) is overlaid on the plots. The ring current electrons (Fig. 2a) and MeV electrons (Fig. 2b) behave very differently in the 

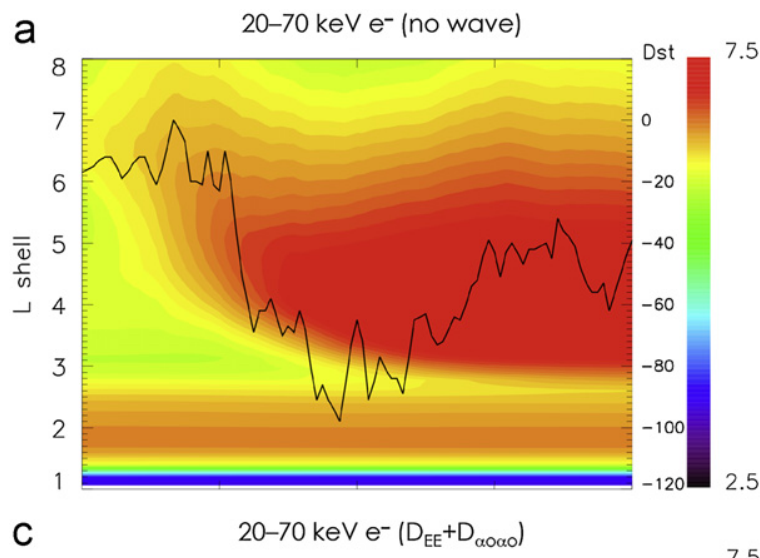

C

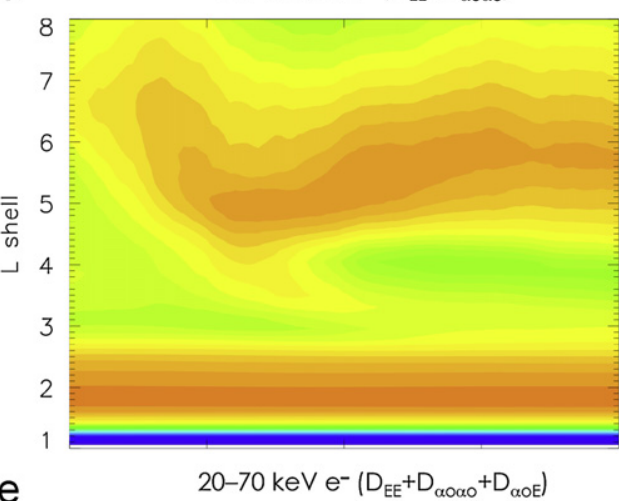

e

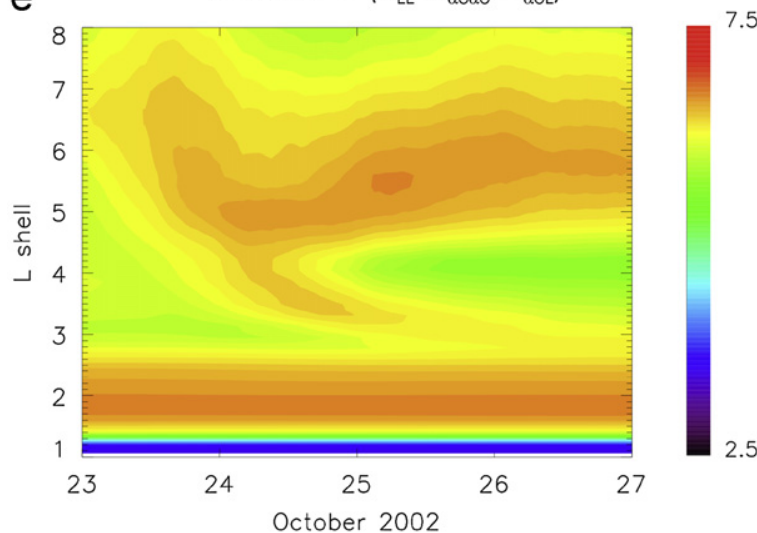

b

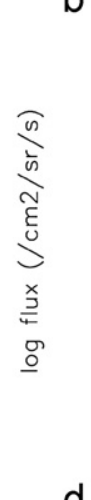

$0.6-1.8 \mathrm{MeV} \mathrm{e}^{-}$(no wave)

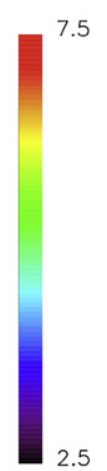

d

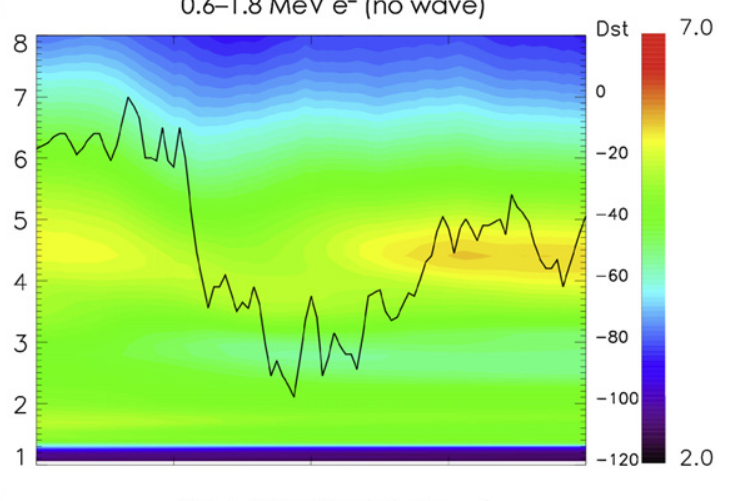

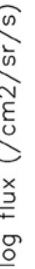
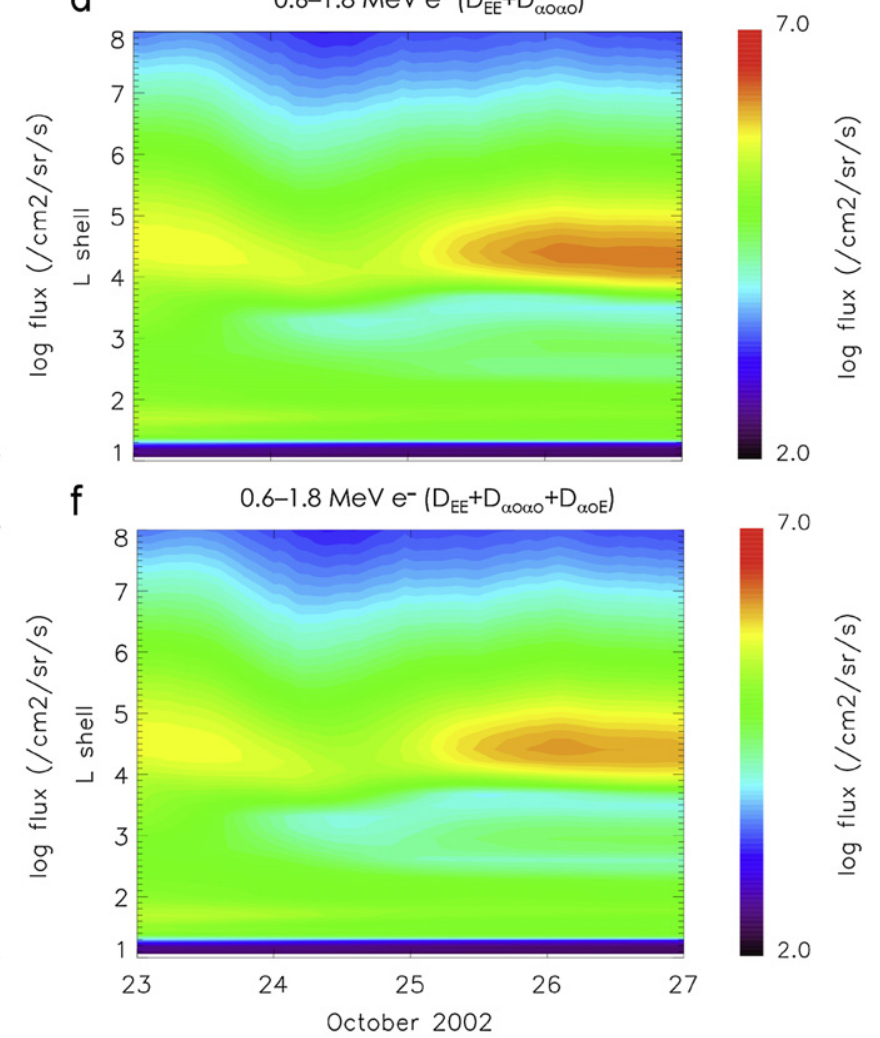

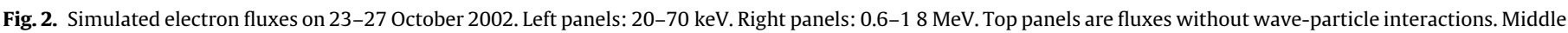
panels are fluxes with energy and pitch-angle diffusion. Bottom panels are fluxes with cross diffusion included. The black curves in the top panels are Dst.

storm. During the main phase, ring current electrons from higher $L$ shells drift earthward and fill the entire outer belt. The fluxes remain high because no wave associated loss mechanism is included in this calculation. For $\mathrm{MeV}$ electrons (Fig. 2b), magnetic field effects dominate over convection. Noticeable flux dropout is seen in the heart of the outer belt during the main phase when ring current is intensified. This is the well known Dst effect (Dessler and Karplus, 1961; Kim and Chan, 1997). The magnetic field produced by the ring current inflates the main field. The drift shells of energetic particles in the radiation belt expand outward correspondingly (conservation of the third adiabatic invariant) and particles decelerate. This deenergization causes an adiabatic decrease in particle fluxes since the energy spectrum slope is negative in the radiation belt energy range. The expansion of drift shells also produces permanent loss at high L's when particles encounter the magnetopause (Ukhorskiy et al., 2006). During the recovery phase, MeV electron fluxes recover as well. The intensities are higher than the pre-storm level.
Fok et al. (2008) found that the flux increase is a result of electron injection and earthward transport during the course of the storm.

Next we examine the effects of wave-particle interactions on the ring current and radiation belt electrons. The middle panels of Fig. 2 show the RBE electron fluxes with pure energy and pitchangle diffusion from interacting with whistler mode chorus. Calculation results with cross diffusion are plotted in the bottom panels. At the beginning of the storm, wave activity is weak. There is no obvious difference between runs with and without chorus waves. During the main and early recovery phases, wave activity is strong. For ring current electrons (left panels), the effects of pitchangle diffusion are stronger than those of energy diffusion. A significant amount of electrons are diffused into the loss cone. A flux hole is formed around $L=4$ during the storm recovery. In contrast, with the consideration of chorus waves, MeV electron fluxes gradually increase during the recovery phase (right panels, Fig. 2). The injection of lower-energy electrons forms a seed 
population for energy diffusion. Particles are accelerated by the chorus waves and $\mathrm{MeV}$ electrons slowly diffuse to larger $L$ shells at late recovery.

When comparing the fluxes in middle panels with those in the bottom panels of Fig. 2, we find noticeable effects of cross diffusion.

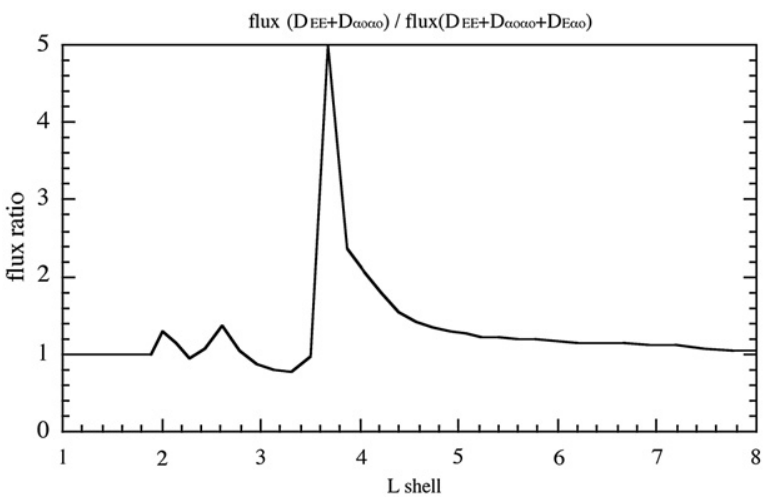

Fig. 3. Flux ratio as a function of time of $\mathrm{MeV}$ electrons without and with cross diffusion. Ratios are calculated at simulation time of 4 days.
Cross diffusion tends to moderate the impacts from pure pitchangle and energy diffusion. For ring current electrons, with cross diffusion included (Fig. 2e), pitch-angle diffusion is weakened and the overall fluxes are higher than those without cross diffusion (Fig. 2c). Similarly in the case of MeV electrons, cross diffusion reduces the energy gain by energy diffusion and thus the flux enhancement in the recovery phase. Fig. 3 illustrates quantitatively the effect of cross diffusion in radiation belt electrons. The ratio of $\mathrm{MeV}$ electron flux without and with cross diffusion is plotted as a function of $L$ shell at the end of 4-days simulation. In most parts of the inner magnetosphere, the ratio is close to 1 . However, in the heart of the outer belt around $L=4$, ignoring cross diffusion could cause overestimation of electron flux as much as a factor of 5 . Detailed analysis of the effects of cross diffusion will be reported in a separate study.

\section{RBE simulation of a MHD substorm}

The Sun was in its deep minimum in years 2008 and 2009; there was minimal solar and geomagnetic activity. However, recurring high speed streams could trigger substorms in the Earth's magnetosphere even in solar minimum (Baker et al., 1998). On 3 September

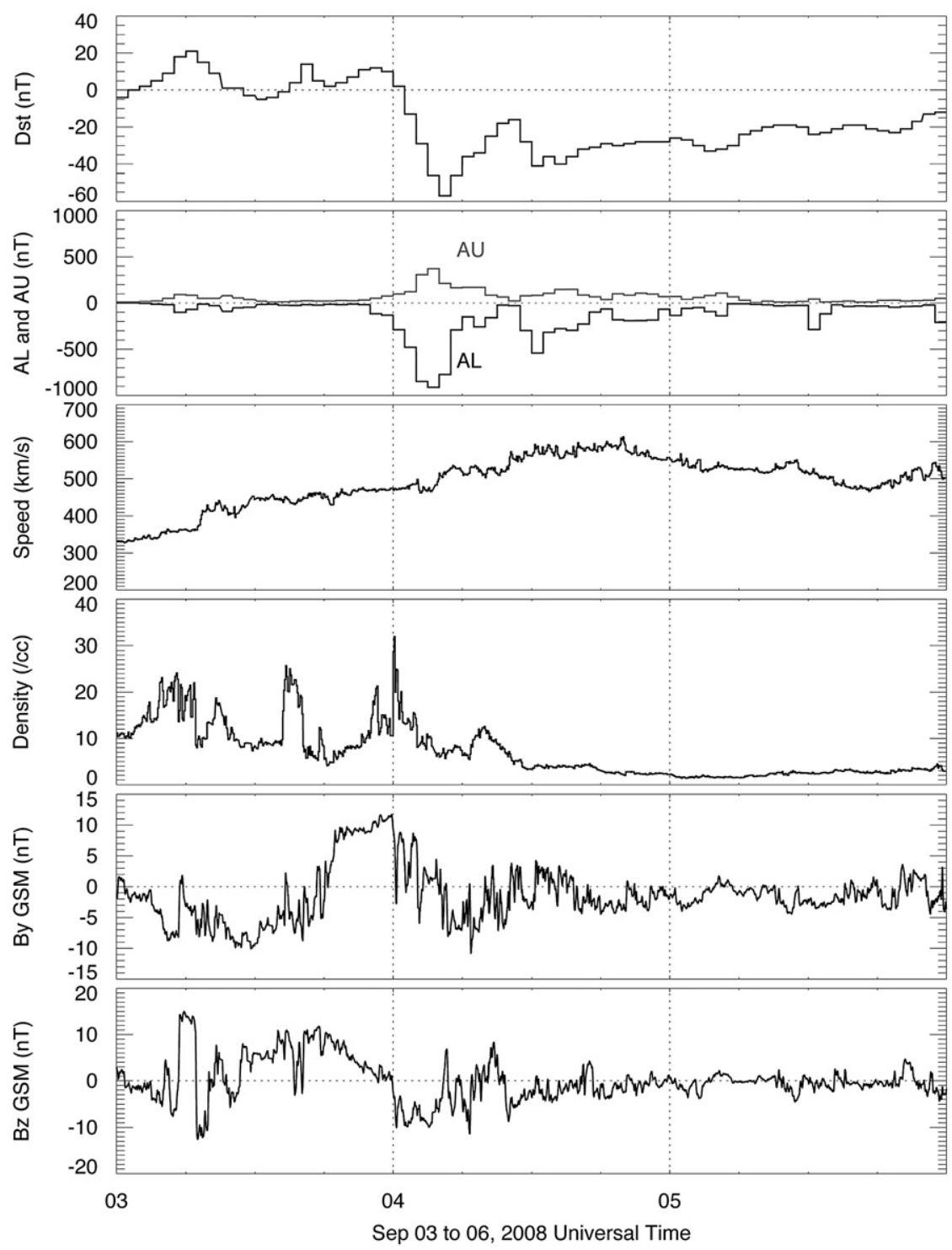

Fig. 4. Dst, AU, AL and solar wind speed, density, By and Bz on 3-6 September 2008. 
a

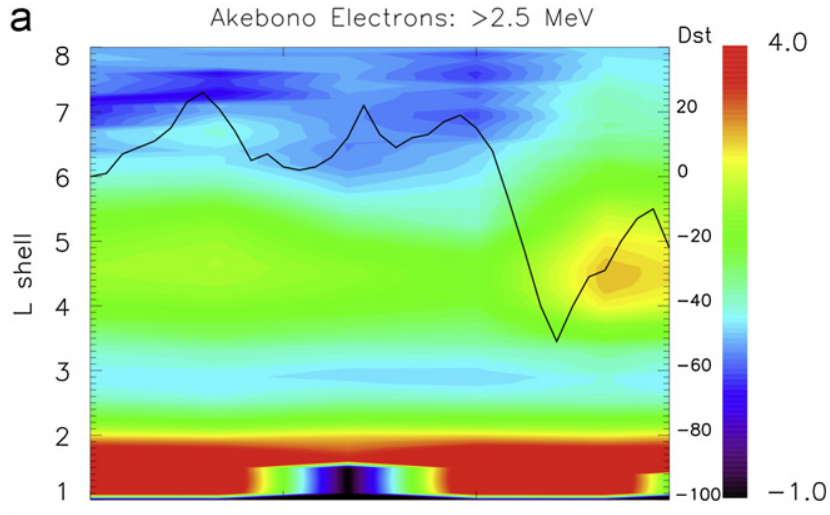

b

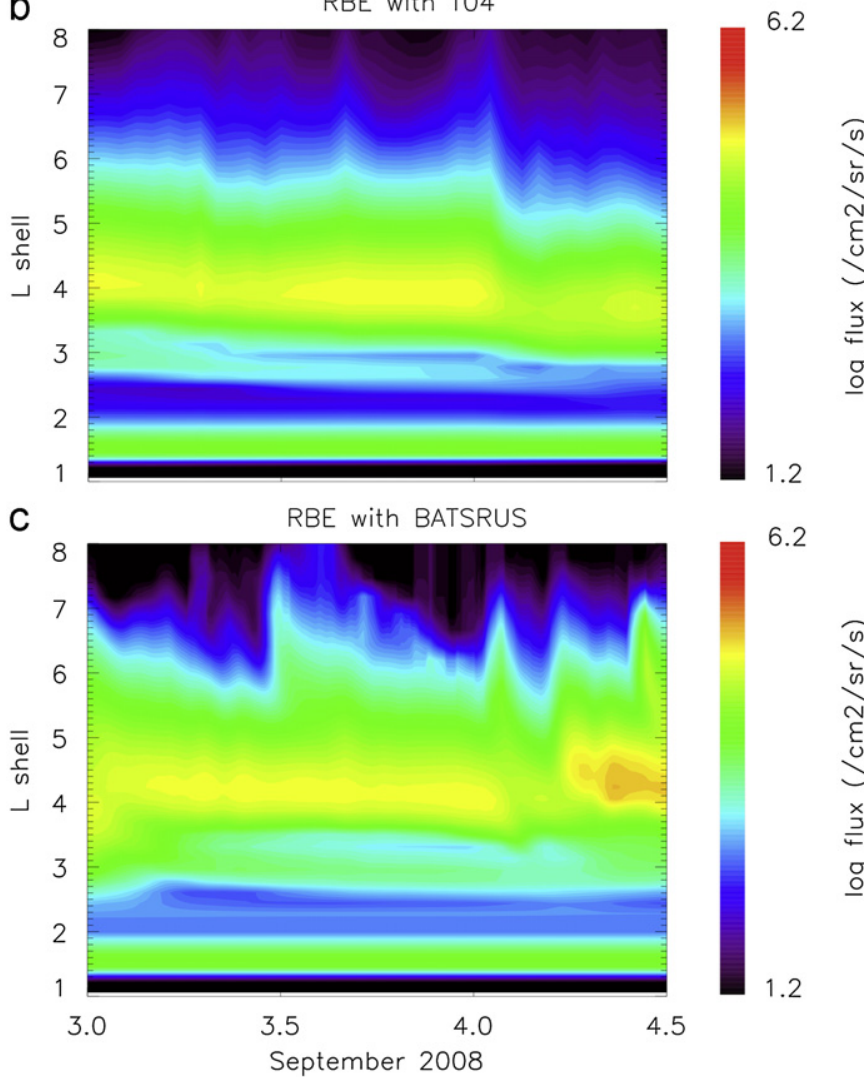

Fig. 5. Top panel: $L$-Time plot of Akebono electron flux $(>2.5 \mathrm{MeV})$ on 3-4 September 2008. The black curve is Dst. Middle panel: corresponding RBE simulate flux with T04 magnetic field. Bottom panel: simulated flux with BATSRUS fields.
2008, a high speed stream arrived at the magnetosphere and triggered a moderate storm with minimum Dst of $-51 \mathrm{nT}$ on September 4, 0500 UT. Fig. 4 plots the Dst, AU, AL and solar wind speed, density, By and Bz on 3-5 September 2008.

The Akebono satellite observed enhancements of radiation belt electrons during the active period on 3-5 September 2008. Akebono was launched in February 1989 by the Institute of Space and Astronautical Science in Japan (Takagi et al., 1993). In September 2008, Akebono was in a high inclination, highly elliptical orbit with apogee at $5260 \mathrm{~km}$ altitude, perigee altitude at $295 \mathrm{~km}$, and orbit period of $2.5 \mathrm{~h}$. The Radiation Monitor (RDM) measured electron fluxes in three energy channels: $0.30-0.95 \mathrm{MeV}$, $0.95-2.5 \mathrm{MeV}$ and $>2.5 \mathrm{MeV}$ (Takagi et al., 1993). A rapid increase in high-energy electron flux with a factor of 80 at $4<L<5$ is seen between 0321 and 0546 UT, around the peak of the storm (minimum Dst). Similar growths are also seen in the other 2 RDM lower-energy channels. The top panel of Fig. 5 shows the Akebono electron flux of energy $>2.5 \mathrm{MeV}$ from September 3, 00 UT to September 4, 12 UT. The Dst index during this time is overlaid in the plot. The Akebono data are averaged over 3 orbit periods. The time scale of this enhancement is a few hours and thus it is too short for wave associated energization. The large AL values in this time period (Fig. 4) suggest a substorm may play a role in this quick increase of outer belt electrons (Nagai et al., 2006).

We perform RBE simulations to understand the cause of the flux enhancement seen in the Akebono electron data. In most of our previous RBE calculations, we used empirical models of magnetic and electric fields (Fok et al., 2001, 2008; Zheng et al., 2003). Recently, we have adapted the magnetic and electric fields output from the coupled model of BATSRUS-Rice Convection Model (RCM) into the RBE model in order to self-consistently simulate the responses of the radiation belts to solar wind and ring current variations during storm time (Glocer et al., 2009). The MHD electromagnetic fields are updated every $10 \mathrm{~s}$ to drive the drift motion and radial transport of radiation belt particles. Fig. 5(b) depicts the RBE electron fluxes calculated with T04 magnetic field model and Weimer electric field model (Tsyganenko et al., 2003; Weimer, 2001). Fig. 5(c) is the RBE flux calculated in the MHD fields simulated from the BATSRUS-RCM model (De Zeeuw et al., 2004). Note that the RBE fluxes shown in Figs. 5(b) and (c) are equatorial fluxes and with temporal resolution of $1 \mathrm{~h}$. In contrast, the Akebono measurements are taken along high inclination orbits. However, the temporal variability of high latitude fluxes was found nearly identical with the equatorial fluxes (Kanekal et al., 2001, 2005). Wave-particle interactions are not included in these RBE calculations. In the quiet period on September 3, the two RBE simulations give similar flux intensity. During the main phase of the storm, both RBE runs produce flux dropout in the outer belt, in

a

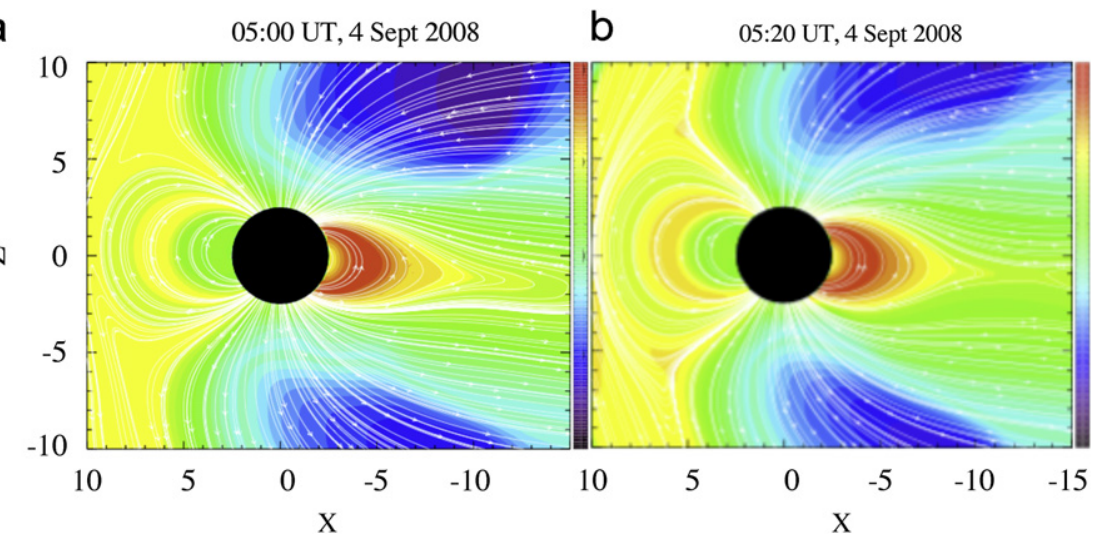

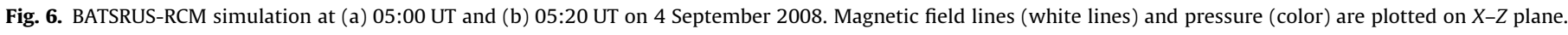


response to the ring current intensification simulated in the T04 and BATSRUS-RCM models. However, at 05-06 UT on September 4, a sudden increase in electron flux is seen at $4<L<5$ in the RBEBATSRUS calculation, consistent with the Akebono data. There is no significant enhancement in the RBE-T04 run during the recovery phase of the storm.

Since wave-particle interactions are not considered in these particular RBE calculations, the enhancement seen in Fig. 5(c) must be a result of particle transport. When we examine the magnetic configuration during the enhancement, we find the MHD model predicts a substorm dipolarization at $\sim 05$ UT on September 4. Fig. 6 shows the BATSRUS field lines in white and pressure in color on the $X-Z$ plane before (left) and after (right) the substorm onset on September 4. At 05:00 UT, the field lines on the nightside are greatly stretched. 20 min later, the dipolarization takes place in the tail. Field lines are convecting earthward and have more dipole-like shape. Electrons which are gyrating along these collapsing field lines can be accelerated significantly on a time scale of minutes (Fok et al., 2001; Glocer et al., 2009), much faster than the time scale for energization by whistler mode chorus waves, which is typically of the order of 1-2 days (Summers and Ma, 2000; Horne et al., 2005). The T04 model, which is driven by Dst and solar wind parameters, does not contain clear substorm signatures. Empirical magnetic field models of this kind cannot directly simulate substorm reconfiguration unless special tricks are applied (Delcourt et al., 1990, 1997; Pulkkinen et al., 1991; Fok et al., 2001). For moderate storms such as this one on 3-5 September 2008, convection is weak and the dominant energization and transport mechanism is substorm reconfiguration and the resulting dipolarization electric field. The rapid enhancements of radiation belt fluxes during modest storms cannot be explained without the consideration of substorm effects.

\section{Discussion and conclusions}

One must be cautious to interpret the timing signature seen in the Akebono data. The temporal resolution is limited by the orbit periods $(2.5 \mathrm{~h})$. In our separate study of the storm in September 2008, we will identify storm and substorm signatures using continuous high resolution data, such as measurements from NOAA and GOES satellites.

So far we have considered only low-latitude whistler mode chorus waves. However, study has shown that chorus waves at high latitude are important in the loss and acceleration of energetic electrons (Horne and Thorne, 2003). Other wave modes, such as plasmaspheric hiss and electromagnetic ion cyclotron waves, also play crucial roles in the development and decay of the radiation belts (Meredith et al., 2007; Lorentzen et al., 2000; Summers and Thorne, 2003). All these wave modes have different sources of excitation and are found in different regions of the inner magnetosphere. We plan to gradually include all the important wave modes in the RBE model. In that case we will be in a better position to understand and identify the physical processes that control the observed variability in the radiation belts.

Inclusion of the cross diffusion term does make a noticeable difference in electron flux in the heart of the outer belt. However, the effect is relatively mild and localized when compared with other processes such as particle injection, transport and acceleration. It is difficult to show quantitatively that with cross diffusion will improve the data model comparison. Nevertheless, inclusion of cross diffusion will give a better estimation of the diffusive effect from interacting with a particular wave mode.

The dynamics of radiation belt electrons is strongly controlled by the magnetic configuration and its fluctuations. The empirical models of Tsygenenko (Tsyganenko and Stern, 1996; Tsyganenko et al., 2003) have been used to simulate the temporal variations of magnetic field in the RBE model. We found strong electron energization by the inductive electric field associated with the time-varying magnetic field (Zheng et al., 2003; Fok et al., 2008). However, as shown in Section 4, we cannot reproduce the rapid flux enhancement during a substorm dipolarization with the T04 model. In contrast, the RBE model driven by MHD fields successfully produces the observed increase in electron flux during a substorm. Another limitation of the combined RBE-T04Weimer models is that they cannot fully simulate the effect of radial diffusion even with T04 and Weimer model input parameters updated in time. Both the Tsyganenko and Weimer models are empirical and not consistent with each other. This problem can be resolved by, again, applying MHD fields in the RBE model. Recently work by Huang et al. (2010) has shown that the ULF waves ( $\mathrm{mHz}$ range) predicted by the Lyon-Fedder-Mobarry MHD model well represent the ULF wave data observed by the GOES satellites.

In summary, we have reported recent developments and improvements in our radiation belt model. We have implemented pitch-angle-energy cross diffusion in our wave diffusion calculation. We have simulated a substorm dipolarization event with the RBE model embedded in MHD magnetic and electric fields. The findings from this model development work include

(1) Cross diffusion moderates the effects of pure pitch-angle and pure energy diffusion. Exclusion of cross diffusion would significantly overestimate the flux enhancements of relativistic electrons during storm recovery. In our simulation of the storm on 23-27 October 2002, at the heart of the outer belt, the overestimation can be as high as a factor of 5 .

(2) The strong inductive field during substorm dipolarization produces rapid increase in energetic electron flux on a time scale of an hour, much shorter than that from wave acceleration.

\section{Acknowledgment}

This research was supported by NASA Science Mission Directorate, Heliophysics Division, Living With a Star Targeted Research and Technology Program, under Work Breakdown Structures: 936723.02.01.06.78 and 936723.02.01.01.27.

\section{Appendix: Finite difference representation of the ADI scheme}

Eq. (6) outlines the ADI scheme in solving the cross diffusion equation. The second order finite difference discretization of (6) can be written as

$$
\begin{aligned}
& \mu_{k, m}^{1} f_{k-1, m}^{n+1 / 2}+f_{k, m}^{n+1 / 2}-\mu_{k, m}^{1} f_{k+1, m}^{n+1 / 2} \\
& =f_{k, m}^{n}+\mu_{k, m}^{2}\left(f_{k, m+1}^{n}-f_{k, m-1}^{n}\right) \\
& \quad+\mu_{k, m}^{3}\left(f_{k+1, m+1}^{n}+f_{k-1, m-1}^{n}-f_{k-1, m+1}^{n}-f_{k+1, m-1}^{n}\right) \\
& \quad \\
& \mu_{k, m}^{2} f_{k, m}^{n+1}+f_{k, m}^{n+1}-\mu_{k, m}^{2} f_{k, m}^{n+1} \\
& =f_{k, m}^{n+1 / 2}+\mu_{k, m}^{1}\left(f_{k+1, m}^{n+1 / 2}-f_{k-1, m}^{n+1 / 2}\right) \\
& \quad+\mu_{k, m}^{3}\left(f_{k+1, m+1}^{n+1 / 2}+f_{k-1, m-1}^{n+1 / 2}-f_{k-1, m+1}^{n+1 / 2}-f_{k+1, m-1}^{n+1 / 2}\right)
\end{aligned}
$$

where

$\mu_{k, m}^{1}=\frac{\Delta t}{2} \frac{G_{k, m+1} \tilde{D}_{k, m+1}-G_{k, m-1} \tilde{D}_{k, m-1}}{G_{k, m}\left(\alpha_{o m+1}-\alpha_{o m-1}\right)\left(\varepsilon_{k+1}-\varepsilon_{k-1}\right)}$ 
$\mu_{k, m}^{2}=\frac{\Delta t}{2} \frac{G_{k+1, m} \tilde{D}_{k+1, m}-G_{k-1, m} \tilde{D}_{k-1, m}}{G_{k, m}\left(\alpha_{o m+1}-\alpha_{o m-1}\right)\left(\varepsilon_{k+1}-\varepsilon_{k-1}\right)}$

$\mu_{k, m}^{3}=\frac{\Delta t \tilde{D}_{k, m}}{\left(\alpha_{o m+1}-\alpha_{o m-1}\right)\left(\varepsilon_{k+1}-\varepsilon_{k-1}\right)}$

$k$ is $\varepsilon$ index and $m$ is $\alpha_{0}$ index.

Eq. (7) represents two tri-diagonal systems similar to the Crank-Nicolson method. There are well established numerical techniques and stability analysis for this type of problem (LeVeque, 2002; Burden and Faires, 2004).

\section{References}

Albert, J.M., 1994. Quasi-linear pitch angle diffusion coefficients: retaining high harmonics. J. Geophys. Res. 99, 23,741-23,745.

Albert, J.M., Young, S.L., 2005. Multidimensional quasi-linear diffusion of radiation belt electrons 32, L14110. doi:10.1029/2005GL023191Geophys. Res. Lett. 32, L14110. doi:10.1029/2005GL023191.

Albert, J.M., Brautigam, D.H., Hilmer, R.V., Ginet, G.P., 2001. Dynamic radiation belt modeling at the Air Force Research Laboratory. In: Song (Ed.), Space Weather Geophysics Monograph Series, vol. 125. AGU, Washington D. C, pp. 281-287.

Albert, J.M., Meredith, N.P., Horne, R.B., 2009. Three-dimensional diffusion simulation of outer radiation belt electrons during the 9 October 1990 magnetic storm. J. Geophys. Res. 114, A09214. doi:10.1029/2009JA014336.

Baker D.N. 2001. Satellite anomalies due to space storms. In: Daglis, I. A. (Ed.), Space Storms and Space Weather Hazards. Kluwer Academic Publishers, Netherlands, pp. 285-311.

Baker, D.N., Pulkkinen, T.I., Li, X., Kanekal, S.G., Blake, J.B., Selesnick, R.S., Henderson, M.G., Reeves, G.D., Spence, H.E., Rostoker, G., 1998. Coronal mass ejections, magnetic clouds, and relativistic magnetospheric electron events: ISTP. J. Geophys. Res. 103 (A8), 17,279-17,291.

Borovsky, J.E., Thomsen, M.F., Elphic, R.C., 1998. The driving of the plasma sheet by the solar wind. J. Geophys. Res. 103, 17,617-17,639.

Bourdarie, S., Boscher, D., Beutier, T., Sauvaud, J.-A., Blanc, M., 1997. Electron and proton radiation belt dynamic simulations during storm periods: a new asymmetric convection-diffusion model. J. Geophys. Res. 102, 17,541-17,552.

Burden, R.L., Faires, J.D., 2004. Numerical Analysis 8th ed. Brooks Cole, Pacific Grove, CA

Degeling, A.W., Ozeke, L.G., Rankin, R., Mann, I.R., Kabin, K., 2008. Drift resonan generation of peaked relativistic electron distributions by Pc 5 ULF waves. J. Geophys. Res. 113, A02208. doi:10.1029/2007JA012411

Delcourt, D.C., Sauvaud, J.A., Pedersen, A., 1990. Dynamics of single particle orbits during substorm expansion phase. J. Geophys. Res. 95, 20,853-20,865.

Delcourt, D.C., Sauvaud, J.-A., Moore, T.E., 1997. Phase bunching during substorm dipolarization. J. Geophys. Res. 102, 24,313-24,324

Dessler, A.J., Karplus, R., 1961. Some effects of diamagnetic ring currents on Van Allen radiation. J. Geophys. Res. 66, 2289-2295.

De Zeeuw, D.L., Sazykin, S., Wolf, R.A., Gombosi, T.I., Ridley, A.J., Toth, G., 2004 Coupling of a global MHD code and an inner magnetospheric model: initial results. J. Geophys. Res. 109, A12219. doi:10.1029/2003JA010366.

Fok, M.-C., Moore, T.E., 1997. Ring current modeling in a realistic magnetic field configuration. Geophys. Res. Lett. 24, 1775-1778.

Fok, M.-C., Kozyra, J.U., Nagy, A.F., Rasmussen, C.E., Khazanov, G.V., 1993. Decay of equatorial ring current ions and associated aeronomical consequences. J. Geophys. Res. 98, 19381-19393.

Fok, M.-C., Moore, T.E., Greenspan, M.E., 1996. Ring current development during storm main phase. J. Geophys. Res. 101, 15,311-15,322.

Fok, M.-C., Moore, T.E., Spjeldvik, W.N., 2001. Rapid enhancement of radiation belt electron fluxes due to substorm dipolarization of the geomagnetic field. J. Geophys. Res. 106, 3873-3881.

Fok, M-C., Ebihara, Y., Moore, T.E. Ober, D.M. Keller, K.A, 2005. Geospace storm processes coupling the ring current, radiation belt and plasmasphere. In: Burch, J. (Ed.), Inner Magnetosphere Interactions: New Perspectives from Imaging Geophys. Monograph Series, vol. 159. AGU, Washington, D. C, pp. 207-220.

Fok, M.-C., Horne, R.B., Meredith, N.P., Glauert, S.A., 2008. The radiation belt environment model: application to space weather nowcasting. J. Geophys. Res. 113, A03S08. doi:10.1029/2007JA012558.

Fung S.F. 1996. Recent development in the NASA trapped radiation model. In: Lemaire, J.F., Heynderickx, D., Baker, D.N. (Eds.), Radiation Belts: Models and Standards, Geophys. Monograph Series, vol. 97. AGU, Washington, D. C, pp. 79-91

Glauert, S.A., Horne, R.B., 2005. Calculation of pitch angle and energy diffusion coefficients with the PADIE code. J. Geophys. Res. 110, A04206. doi:10.1029/ 2004JA010851

Glocer, A., Toth, G., Fok, M., Gombosi, T., Liemohn, M., 2009. Integration of the radiation belt environment model into the space weather modeling framework J. Atmos. Sol. Terr. Phys.. doi:10.1016/j.jastp.2009.01.003.

Green, J.C., Onsager, T.G., O’Brien, T.P., Baker, D.N., 2004. Testing loss mechanisms capable of rapidly depleting relativistic electron flux in the Earth's outer radiation belt. J. Geophys. Res. 109, A12211 doi:10.1029/2004JA010579.

Horne, R.B., 2002. The contribution of wave particle interactions to electron loss and acceleration in the Earth's radiation belts during geomagnetic storms. In: Stone, W.R. (Ed.), Review of Radio Science 1999-2002. John Wiley, Bognor Regis, pp. 801-828 Chapter 33.
Horne, R.B., Thorne, R.M., 1998. Potential waves for relativistic electron scattering and stochastic acceleration during magnetic storms. Geophys. Res. Lett. 25, 3011.

Horne, R.B., Thorne, R.M., 2003. Relativistic electron acceleration and precipitation during resonant interactions with whistler-mode chorus. Geophys. Res. Lett. 30 (10), 1527. doi:10.1029/2003GL016973.

Horne, R.B., Thorne, R.M., Glauert, S.A., Albert, J.M., Meredith, N.P., Anderson, R.R., 2005. Timescale for radiation belt electron acceleration by whistler mode chorus waves. J. Geophys. Res. 110, A03225. doi:10.1029/2004JA010811.

Horne, R.B., Meredith, N.P., Glauert, S.A., Varotsou, A., Boscher, D., Thorne, R.M., Shprits, Y.Y., Anderson, R.R., 2006. Mechanisms for the acceleration of radiation belt electrons. In: Tsurutani, B.T. (Ed.), Recurrent Magnetic Storms: Corotating Solar Wind Streams, Geophysics Monograph Series, vol. 167. AGU, Washington, D. C, pp. 151-173.

Horne, R.B., Thorne, R.M., Glauert, S.A., Meredith, N.P., Pokhotelov, D., Santolik, O., 2007. Electron acceleration in the Van Allen radiation belts by fast magnetosonic waves. Geophys. Res. Lett. 34, L17107. doi:10.1029/2007GL030267.

Huang, C.-L., Spence, H.E., Singer, H.J., Hughes, W.J., 2010. Modeling radiation radial diffusion in ULF wave fields: 1 . Quantifying ULF wave power at geosynchronous orbit in observations and in global MHD model. J. Geophys. Res. 115, A06215. doi:10.1029/2009JA014917.

Iles, R.H.A., Fazakerley, A.N., Johnstone, A.D., Meredith, N.P., Buhler, P., 2002. The relativistic electron response in the outer radiation belt during magnetic storms. Ann. Geophys. 20, 957-965.

Kanekal, S.G., Baker, D.N., Blake, J.B., 2001. Multisatellite measurements of relativistic electron: global coherence. J. Geophys. Res. 106, 29,721-29,732.

Kanekal, S.G., Friedel, R., Reeves, G.D., Baker, D.N., Blake, J.B., 2005. Relativistic electron events in 2002: Studies of pitch angle isotropization. J. Geophys. Res. 110, A12224. doi:10.1029/2004JA010974.

Kim, H.-J., Chan, A.A., 1997. Fully relativistic changes in storm time relativistic electron fluxes. J. Geophys. Res. 102, 22107-22116.

LeVeque, R.J., 2002. Finite volume methods for hyperbolic problems. Cambridge University Press, New York.

Li, X., Temerin, M., Baker, D.N., Reeves, G.D., Larson, D., 2001. Quantitative prediction of radiation belt electrons at geostationary orbit based on solar wind measurements. Geophys. Res. Lett. 28, 1887-1890.

Lorentzen, K.R., McCarthy, M.P., Parks, G.K., Foat, J.E., Millan, R.M., Smith, D.M., Lin, R.P., Treilhou, J.P., 2000. Precipitation of relativistic electrons by interaction with electromagnetic ion cyclotron waves. J. Geophys. Res. 105, 5381-5389.

Lyons, L.R., 1973. Comments on pitch angle diffusion in the radiation belts. J. Geophys. Res. 78, 6793-6797.

Lyons, L.R., Thorne, R.M., Kennel, C.F., 1972. Pitch-angle diffusion of radiation belt electrons with the plasmasphere. J. Geophys. Res. 77, 3455-3474.

Meredith, P.N., Horne, R.B., Anderson, R.R., 2001. Substorm dependence of chorus amplitudes: implications for the acceleration of electrons to relativistic energy. J. Geophys. Res. 106, 13,165-13,178.

Meredith, P.N., Horne, R.B., Thorne, R.M., Anderson, R.R., 2003. Favored regions for chorus-driven electron acceleration to relativistic energies in the Earth's outer radiation belt. Geophys. Res. Lett. 30 (16), 1871

Meredith, N.P., Horne, R.B., Glauert, S.A., Anderson, R.R., 2007. Slot region electron loss timescales due to plasmaspheric hiss and lightning generated whistlers. J. Geophys. Res. 112, A08214. doi:10.1029/2007JA012413.

Miyoshi, Y.S., Jordanova, V.K., Morioka, A., Thomsen, M.F., Reeves, G.D., Evans, D.S. Green, J.C., 2006. Observations and modeling of energetic electron dynamics during the October 2001 storm. J. Geophys. Res. 111, A11S02. doi: 10.1029/2005JA011351.

Nagai, T., Yukimatu, A.S., Matsuoka, A., Asai, K.T., Green, J.C., Onsager, T.G., Singer, H.J. 2006. Timescales of relativistic electron enhancements in the slot region. J. Geophys. Res. 111, A11205. doi:10.1029/2006JA011837.

Ober, D.M., Horwitz, J.L., Gallagher, D.L., 1997. Formation of density troughs embedded in the outer plasmasphere by subauroral ion drift events. J. Geophys. Res. 102, 14,595-14,602.

Omura, Y., Summers, D., 2006. Dynamics of highenergy electrons interacting with whistler mode chorus emissions in the magnetosphere. J. Geophys. Res. 111, A09222. doi:10.1029/2006JA011600.

Paulikas, G.A., Blake, J.B., 1979. Effects of the solar wind on magnetospheric dynamics: energetic electrons at the synchronous orbit. In: Olsen, W.-P. (Ed.), Quantitative Modeling of Magnetospheric Processes, vol. 21. AGU, Washington, D. C, pp. $180-202$

Pulkkinen, T.I., Baker, D.N., Fairfield, D.H., Pellinen, R.J., Murphree, J.S., Elphinstone, R.D., McPherron, R.L., Fennell, J.F., Lopez, R.E., Nagai, T., 1991. Modeling the growth phase of a substorm using the Tsyganenko model and multi-spacecraft observations: CDAW-9. Geophys. Res. Lett. 18, 1963-1966.

Reeves, G.D., McAdams, K.L., Friedel, R.H.W., 2003. Acceleration and loss of relativistic electrons during geomagnetic storms. Geophys. Res. Lett. 30, 1529.

Schulz, M., Lanzerotti, L.J., 1974. Physics and chemistry in space. Particle Diffusion in the Radiation Belts, vol. 7. Springer, New York.

Shprits, Y.Y., Chen, L., Thorne, R.M., 2009. Simulations of pitch angle scattering of relativistic electrons with MLT-dependent diffusion coefficients. J. Geophys. Res. 114, A03219. doi:10.1029/2008JA013695.

Summers, D., Ma, C.-Y., 2000. A model for generating relativistic electrons in the Earth's inner magnetosphere based on gyroresonant wave-particle interactions. J. Geophys. Res. 105, 2625-2639.

Summers, D., Thorne, R.M., 2003. Relativistic electron pitch-angle scattering by electromagnetic ion cyclotron waves during geomagnetic storms. J. Geophys. Res. 108 (A4), 1143. 
Summers, D., Thorne, R.M., Xiao, F., 1998. Relativistic theory of wave-particle resonant diffusion with application to electron acceleration in the magnetosphere. J. Geophys. Res. 103, 20,487-20,500.

Summers, D., Ma, C., Mukai, T., 2004. Competition between acceleration and loss mechanisms of relativistic electrons during geomagnetic storms. J. Geophys. Res. 109, A04221. doi:10.1029/2004JA010437.

Summers, D., Ni, B., Meredith, N.P., 2007. Timescales for radiation belt electron acceleration and loss due to resonant wave particle interactions: 2. Evaluation for VLF chorus, ELF hiss and EMIC waves. J. Geophys. Res. 112, A04207. doi:10.1029/2006JA011993.

Takagi, S., Nakamura, T., Kohno, T., Shiono, N., Makino, F., 1993. Observation of space radiation environment with EXOS-D. IEEE Trans. Nucl. Sci. 40, 1491-1497.

Tao, X., Chan, A.A., Albert, J.M., Miller, J.A., 2008. Stochastic modeling of multidimensional diffusion in the radiation belts. J. Geophys. Res. 113, A07212. doi:10.1029/2007JA012985.

Thorne, R.M., Horne, R.B., Glauert, S.A., Meredith, N.P., Shprits, Y.Y., Summers, D., Anderson, R.R., 2005. The influence of wave-particle interactions on relativistic electrons during storms. In: Burch, J., Schulz, M., Spence, H. (Eds.), Inner Magnetosphere Interactions: New Perspectives From Imaging, Geophysics Monograph Series, vol. 159. AGU, Washington D.C.

Tsyganenko, N.A., Stern, D.P., 1996. Modeling the global magnetic field of the largescale Birkeland current systems. J. Geophys. Res. 101, 27,187-27,198.
Tsyganenko, N.A., Singer, H.J., Kasper, J.C., 2003. Storm-time distortion of the inner magnetosphere: How severe can it get? J. Geophys. Res. 108 (A5) 1209. doi:10.1029/2002JA009808.

Ukhorskiy, A., Sitnov, M.I., 2008. Radial transport in the outer radiation belt due to global magnetospheric compressions. J. Atmos. Sol. Terr. Phys. 70, 1714-1726.

Ukhorskiy, A.Y., Anderson, B.J., Brandt, P.C., Tsyganenko, N.A., 2006. Storm time evolution of the outer radiation belt: transport and losses. J. Geophys. Res. 111, A11S03. doi:10.1029/2006JA011690.

Varotsou, A., Boscher, D., Bourdarie, S., Horne, R.B., Glauert, S.A., Meredith, N.P., 2005 Simulation of the outer radiation belt electrons near geosynchronous orbit including both radial diffusion and resonant interaction with whistler mode chorus waves. Geophys. Res. Lett. 32, L19106. doi:10.1029/2005GL023282.

Vette, J.I., The AE-8 trapped electron model environment, NSSDC/WDC-A-R\&S 91 24. In: Proceedings of the NASA Goddard space flight center, Greenbelt, Maryland, November, 1991.

Weimer, D.R., 2001. An improved model of ionospheric electric potentials including substorm perturbations and applications to the Geospace Environment Modeling November 24, 1996, event. J. Geophys. Res. 106, 407-416.

Xiao, F., Su, Z., Zheng, H., Wang, S., 2009. Modeling of outer radiation belt electrons by multidimensional diffusion process. J. Geophys. Res. 114, A03201. doi:10. 1029/2008JA013580.

Zheng, Y., Fok, M.-C., Khazanov, G.V., 2003. A radiation belt-ring current forecasting model. Space Weather 1 (3), 1013. 